\title{
New fractional inequalities of midpoint type via s-convexity and their application
}

\section{Ohud Almutairi ${ }^{1}$ and Adem Kılıçman ${ }^{2 *}$ (D)}

\section{"Correspondence:}

akilic@upm.edu.my

${ }^{2}$ Department of Mathematics and Institute for Mathematical Research Universiti Putra Malaysia, Serdang, Malaysia

Full list of author information is available at the end of the article

\begin{abstract}
In this study, we introduced new integral inequalities of Hermite-Hadamard type via s-convexity and studied their properties. The absolute form of the first and second derivatives for the new inequalities is considered to be s-convex. As an application, the inequalities were applied to the special means of real numbers. We give the error estimates for the midpoint formula.
\end{abstract}

Keywords: Convex functions; Hermite-Hadamard inequality; Hölder's inequality; Special means; Midpoint formula

\section{Introduction}

Due to the significant roles played by convex functions, they exist in many fields of studies including life and management sciences, engineering and optimization [1, 2]. For example, extended and generalized classical convexities are frequently applied in mathematics and optimization inequalities. The theory of convexity is linked with that of inequalities. Therefore, many existing inequalities, reported in the literature, emerged from convex functions [3-5]. One of such fundamental inequalities that have been widely reported in the literature is of the Hermite-Hadamard type. We consider a function $\psi: E \subseteq \mathbb{R} \rightarrow \mathbb{R}$, $u, v \in E$ with $u \leq v$, convex if and only if,

$$
\psi\left(\frac{u+v}{2}\right) \leq \frac{1}{v-u} \int_{u}^{v} \psi(x) d x \leq \frac{\psi(u)+\psi(v)}{2} .
$$

The inequality (1) is most celebrated since it is broadly used in optimization and probability. The complete historical overview of this inequality is given in [6, 7].

Recently, a wide class of inequalities derived through convexity has been studied. This includes the work of Hudzik and Maligranda [8], who rigorously studied the kinds of $s$ convex functions, whose definition is given as follows.

Definition $1 \psi:[0, \infty) \rightarrow \mathbb{R}$ is said to be $s$-convex in the second sense if the inequality

$$
\psi(\vartheta u+(1-\vartheta) v) \leq \vartheta^{s} \psi(u)+(1-\vartheta)^{s} \psi(v)
$$

holds for all $u, v \in[0, \infty), \vartheta \in[0,1]$, and for some fixed $0<s \leq 1$.

(c) The Author(s) 2019. This article is distributed under the terms of the Creative Commons Attribution 4.0 International License (http://creativecommons.org/licenses/by/4.0/), which permits unrestricted use, distribution, and reproduction in any medium, provided you give appropriate credit to the original author(s) and the source, provide a link to the Creative Commons license, and indicate if changes were made. 
This inequality, representing a second sense of $s$-convexity, is often represented by $K_{s}^{2}$. When $s=1$, the s-convexity remains as for the ordinary function on the interval $[0, \infty)$.

Given the generalization of inequalities, Dragomir and Fitzpatrick studied a HermiteHadamard type in the second sense for an s-convex function [3].

Theorem 1 Let $\psi:[0, \infty) \rightarrow[0, \infty)$ be an s-convex function in the second sense, whereby $s \in(0,1]$, and $u, v \in[0, \infty), u<v$. If $\psi \in L^{1}[0,1]$, the following inequalities hold:

$$
2^{s-1} \psi\left(\frac{u+v}{2}\right) \leq \frac{1}{v-u} \int_{u}^{v} \psi(x) d x \leq \frac{\psi(u)+\psi(v)}{s+1}
$$

The constant $\Lambda=\frac{1}{s+1}$ makes the above inequality sharp, which is the best possible in the second inequality in (3).

The two inequalities for differentiable convex mapping of Hadamard type were introduced by Dragomir and Agarwal [9]; the results can be proved by applying the following lemma.

Lemma 1 Suppose $\psi: E \subseteq \mathbb{R} \rightarrow \mathbb{R}$ is a differentiable mapping on $E^{\circ}$, and $u, v \in E$ with $u \leq v$, then we have

$$
\frac{\psi(u)+\psi(v)}{2}-\frac{1}{v-u} \int_{u}^{v} \psi(x) d x=\frac{v-u}{2} \int_{0}^{1}(1-t) \psi^{\prime}(t u+(1-t) v) d t .
$$

Here, $E^{\circ}$ indicates the interior of $E$.

The other general result connected to (4) was studied in [10]. The formula given in the next lemma can be found in [3].

Lemma 2 Suppose $\psi: E \subseteq \mathbb{R} \rightarrow \mathbb{R}$ is a differentiable mapping on $E^{\circ}$, and $u, v \in I$ with $u \leq v$, we have

$$
\begin{aligned}
& \frac{1}{v-u} \int_{u}^{v} \psi(x) d x-\psi\left(\frac{u+v}{2}\right) \\
& \quad=(v-u)\left[\int_{0}^{\frac{1}{2}} \vartheta \psi^{\prime}(v+(u-v) \vartheta) d t+\int_{\frac{1}{2}}^{1}(t-1) \psi^{\prime}(v+(u-v) t) d t\right] .
\end{aligned}
$$

In [5], Dragomir and Pearce established Hadamard-type inequalities for differentiable convex mapping. The following lemma was used to derive the inequalities.

Lemma 3 Let $\psi: I \subseteq \mathbb{R} \rightarrow \mathbb{R}$ be a differentiable mapping on $E^{\circ}$, and $u, v \in I$ with $u \leq v$, we have

$$
\frac{\psi(u)+\psi(v)}{2}-\frac{1}{v-u} \int_{u}^{v} \psi(x) d x=\frac{(v-u)^{2}}{2} \int_{0}^{1} \vartheta(1-t) \psi^{\prime \prime}(t u+(1-t) v) d t
$$

The generalization of inequality (1) for different convexity, such as $s$-convex, $r$-convex and $m$-convex functions, have been studied by a number of mathematicians. The theorem on how some of the convex functions are formulated is given by Kirmaci in [11]. In [12], the authors introduced a new improvement of the Hermite-Hadamard inequality on a coordinated convex function. 
In [13], a presumably new Hermite-Hadamard-type inequality was established by Mehrez and Agarwal, whose finding is reported in the next theorem.

Theorem 2 Suppose that $\psi: E^{\circ} \subset \mathbb{R} \rightarrow \mathbb{R}$ is a differentiable mapping on $E^{\circ}, u, v \in E^{\circ}$ with $u<v$. Let the derivative of $\psi$ be $\psi^{\prime}:\left[\frac{3 u-v}{2}, \frac{3 v-u}{2}\right] \rightarrow \mathbb{R}$, a continuous function on $\left[\frac{3 u-v}{2}, \frac{3 v-u}{2}\right]$. Let $q \geq 1$, if $\left|\psi^{\prime}\right|$ is convex on $\left[\frac{3 u-v}{2}, \frac{3 v-u}{2}\right]$, then we have the following:

$$
\left|\frac{1}{v-u} \int_{u}^{v} \psi(x) d x-\psi\left(\frac{u+v}{2}\right)\right| \leq \frac{v-u}{8}\left(\left|\psi^{\prime}\left(\frac{3 u-v}{2}\right)\right|^{q}+\left|\psi^{\prime}\left(\frac{3 u-v}{2}\right)\right|^{q}\right)^{\frac{1}{q}} .
$$

This paper is aimed at establishing some new integral inequalities for s-convexity linked with (1). Even though the newly established inequalities are generalized forms of Theorem 2, they can be of the form of a class function with first and second derivatives for $s$-convexity in the second sense. As an application, the inequalities for special means are derived. Error estimates for the midpoint formula are also studied here.

\section{Main results}

The main result can be confirmed by applying the next lemma.

Lemma 4 Suppose that $\psi: E \subseteq \mathbb{R} \rightarrow \mathbb{R}$ is a differentiable mapping on $E^{\circ}$, and $u, v \in E$ with $u \leq v$. If $\psi$ is an $s$-convex function whereby $s \in(0,1]$, we obtain the following:

$$
2^{s-1} \psi\left(\frac{u+v}{2}\right) \leq \frac{1}{v-u} \int_{u}^{v} \psi(x) d x \leq \frac{(s+1) \psi\left(\frac{u+v}{2}\right)+\psi\left(\frac{3 u-v}{2}\right)+\psi\left(\frac{3 v-u}{2}\right)}{2(s+1)}
$$

and

$$
\left|\frac{1}{v-u} \int_{u}^{v} \psi(x) d x-\frac{\psi\left(\frac{u+v}{2}\right)}{2}\right| \leq\left|\frac{\psi\left(\frac{3 u-v}{2}\right)+\psi\left(\frac{3 v-u}{2}\right)}{2(s+1)}\right|
$$

Proof By changing the variable $x=\frac{3}{4} t+\frac{u+v}{4}$, where $t \in\left[\frac{3 u-v}{2}, \frac{3 v-u}{2}\right]$, we obtain

$$
\begin{aligned}
\frac{1}{v-u} \int_{u}^{v} \psi(x) d x & =\frac{3}{4(v-u)} \int_{\frac{3 u-v}{3}}^{\frac{3 v-u}{3}} \psi\left(\frac{3}{4} t+\frac{u+v}{4}\right) d t \\
& \left.\leq \frac{3}{8(v-u)} \int_{\frac{3 u-v}{3}}^{\frac{3 v-u}{3}} \psi\left(\frac{3}{2} t\right)+\psi\left(\frac{u+v}{2}\right)\right] d t \\
& =\frac{\psi\left(\frac{u+v}{2}\right)}{2}+\frac{1}{4(v-u)} \int_{\frac{3 u-v}{2}}^{\frac{3 v-u}{2}} \psi(t) d t
\end{aligned}
$$

which is similar to that reported in [13].

Using the second and third parts of inequality (3), we get

$$
\frac{1}{4(v-u)} \int_{\frac{3 u-v}{2}}^{\frac{3 v-u}{2}} \psi(t) d t \leq \frac{\psi\left(\frac{3 u-v}{2}\right)+\psi\left(\frac{3 v-u}{2}\right)}{2(s+1)}
$$

Considering (8) and (9), the proof of inequalities (6) and (7) is complete. 
Applying the following theorem, we obtained a new result of Hermite-Hadamard-type inequality for $s$-convexity.

Theorem 3 Suppose that $\psi: E^{\circ} \subset \mathbb{R} \rightarrow \mathbb{R}$ is a differentiable mapping on $E^{\circ}, u, v \in E^{\circ}$ with $u<v$. Let the derivative of $\psi$ be $\psi^{\prime}:\left[\frac{3 u-v}{2}, \frac{3 v-u}{2}\right] \rightarrow \mathbb{R}$, a continuous function on $\left[\frac{3 u-v}{2}, \frac{3 v-u}{2}\right]$. Let $q \geq 1$, if $\left|\psi^{\prime}\right|^{q}$ is an s-convex function on $\left[\frac{3 u-v}{2}, \frac{3 v-u}{2}\right]$, for some fixed $s \in(0,1)$, then we have the following:

$$
\begin{aligned}
& \left|\frac{1}{v-u} \int_{u}^{v} \psi(x) d x-\psi\left(\frac{u+v}{2}\right)\right| \\
& \quad \leq(v-u)\left(\frac{1}{8}\right)^{\frac{q-1}{q}}\left[\frac{2-2^{-s}}{2(s+1)(s+2)}\right]^{\frac{1}{q}}\left[\left|\psi^{\prime}\left(\frac{3 u-v}{2}\right)\right|^{q}+\left|\psi^{\prime}\left(\frac{3 v-u}{2}\right)\right|^{q}\right]^{\frac{1}{q}} .
\end{aligned}
$$

Proof Using Lemma 2, we have

$$
\begin{aligned}
\frac{1}{2(v-u)} \int_{\frac{3 u-v}{2}}^{\frac{3 v-u}{2}} \psi(t) d t= & \psi\left(\frac{u+v}{2}\right)+2(v-u)\left[\int_{0}^{\frac{1}{2}} t \psi^{\prime}\left(\frac{3 v-u}{2}+2(u-v) t\right) d t\right. \\
& \left.+\int_{\frac{1}{2}}^{1}(t-1) \psi^{\prime}\left(\frac{3 v-u}{2}+2(u-v) t\right) d t\right]
\end{aligned}
$$

From (8) and (11), we get

$$
\begin{aligned}
\frac{1}{v-u} \int_{u}^{v} \psi(x) d x-\psi\left(\frac{u+v}{2}\right) \leq & (v-u)\left[\int_{0}^{\frac{1}{2}} t \psi^{\prime}\left(\frac{3 v-u}{2}+2(u-v) t\right) d t\right. \\
& \left.+\int_{\frac{1}{2}}^{1}(t-1) \psi^{\prime}\left(\frac{3 v-u}{2}+2(u-v) t\right) d t\right]
\end{aligned}
$$

In the first case, we take $q=1$. We know that $\left|\psi^{\prime}\right|$ is $s$-convex on $\left[\frac{3 u-v}{2}, \frac{3 v-u}{2}\right]$, and for any $t \in[0,1]$ we obtain

$$
\left|\psi^{\prime}\left(t\left(\frac{3 u-v}{2}\right)+(1-t) \frac{3 v-u}{2}\right)\right| \leq t^{s}\left|\psi^{\prime}\left(\frac{3 u-v}{2}\right)\right|+(1-t)^{s}\left|\psi^{\prime}\left(\frac{3 v-u}{2}\right)\right| .
$$

Therefore,

$$
\begin{aligned}
& \int_{0}^{\frac{1}{2}} t \mid \psi^{\prime}\left(\frac{3 v-u}{2}+2(u-v) t \mid d t\right. \\
& \quad=\int_{0}^{\frac{1}{2}} t\left|\psi^{\prime}\left(t\left(\frac{3 u-v}{2}\right)+(1-t)\left(\frac{3 v-u}{2}\right)\right)\right| \\
& \quad \leq \int_{0}^{\frac{1}{2}} t\left[t^{s}\left|\psi^{\prime}\left(\frac{3 u-v}{2}\right)\right|+(1-t)^{s}\left|\psi^{\prime}\left(\frac{3 v-u}{2}\right)\right|\right] d t \\
& \leq\left|\psi^{\prime}\left(\frac{3 u-v}{2}\right)\right| \int_{0}^{\frac{1}{2}} t^{s+1} d t+\left|\psi^{\prime}\left(\frac{3 v-u}{2}\right)\right| \int_{0}^{\frac{1}{2}} t(1-t)^{s} d t \\
& \leq\left[\frac{1}{2^{s+2}(s+2)}\right]\left|\psi^{\prime}\left(\frac{3 u-v}{2}\right)\right|+\left[\frac{4-s 2^{-s}-3 \cdot 2^{-s}}{4((s+1)(s+2))}\right]\left|\psi^{\prime}\left(\frac{3 v-u}{2}\right)\right|,
\end{aligned}
$$


Almutairi and Kılıçman Journal of Inequalities and Applications

(2019) 2019:267

Page 5 of 19

where

$$
\begin{aligned}
& \int_{0}^{\frac{1}{2}} t^{s+1} d t=\frac{1}{2^{s+2}(s+2)} \\
& \int_{0}^{\frac{1}{2}} t(1-t)^{s} d t=\frac{4-s 2^{-s}-3 \cdot 2^{-s}}{4((s+1)(s+2))} .
\end{aligned}
$$

Analogously,

$$
\begin{aligned}
& \int_{\frac{1}{2}}^{1}(1-t) \mid \psi^{\prime}\left(\frac{3 v-u}{2}+2(u-v) t \mid d t\right. \\
& \quad=\int_{\frac{1}{2}}^{1}(1-t)\left|\psi^{\prime}\left(t\left(\frac{3 u-v}{2}\right)+(1-t)\left(\frac{3 v-u}{2}\right)\right)\right| \\
& \quad \leq \int_{\frac{1}{2}}^{1}(1-t)\left[t^{s}\left|\psi^{\prime}\left(\frac{3 u-v}{2}\right)\right|+(1-t)^{s}\left|\psi^{\prime}\left(\frac{3 v-u}{2}\right)\right|\right] d t \\
& \quad \leq\left|\psi^{\prime}\left(\frac{3 u-v}{2}\right)\right| \int_{\frac{1}{2}}^{1} t^{s}(1-t) d t+\left|\psi^{\prime}\left(\frac{3 v-u}{2}\right)\right| \int_{\frac{1}{2}}^{1}(1-t)(1-t)^{s} d t \\
& \quad \leq\left|\psi^{\prime}\left(\frac{3 u-v}{2}\right)\right|\left[\frac{4-s 2^{-s}-3 \cdot 2^{-s}}{4((s+1)(s+2))}\right]+\left|\psi^{\prime}\left(\frac{3 v-u}{2}\right)\right|\left[\frac{1}{2^{s+2}(s+2)}\right]
\end{aligned}
$$

where

$$
\begin{aligned}
& \int_{\frac{1}{2}}^{1} t^{s}(1-t) d t=\frac{4-s 2^{-s}-3 \cdot 2^{-s}}{4((s+1)(s+2))} \\
& \int_{\frac{1}{2}}^{1}(1-t)(1-t)^{s} d t=\frac{1}{2^{s+2}(s+2)} .
\end{aligned}
$$

Thus, from the inequalities (13) and (14), we obtain

$$
\begin{aligned}
& \left|\frac{1}{v-u} \int_{u}^{v} \psi(x) d x-\psi\left(\frac{u+v}{2}\right)\right| \\
& \quad \leq(v-u)\left[\frac{2-2^{-s}}{2(s+1)(s+2)}\right]\left[\left|\psi^{\prime}\left(\frac{3 u-v}{2}\right)\right|+\left|\psi^{\prime}\left(\frac{3 v-u}{2}\right)\right|\right] .
\end{aligned}
$$

Now in the second case, taking $p=\frac{q}{q-1}$, when $q>1$, we apply the Hölder inequality as follows:

$$
\begin{aligned}
& \int_{0}^{\frac{1}{2}} t\left|\psi^{\prime}\left(\frac{3 v-u}{2}+2(u-v) t\right)\right| d t \\
& \quad=\int_{0}^{\frac{1}{2}} t\left|\psi^{\prime}\left(t\left(\frac{3 u-v}{2}\right)+(1-t)\left(\frac{3 v-u}{2}\right)\right)\right| \\
& =\int_{0}^{\frac{1}{2}} t^{1-\frac{1}{q}}\left[t^{\frac{1}{q}}\left|\psi^{\prime}\left(t\left(\frac{3 u-v}{2}\right)+(1-t)\left(\frac{3 v-u}{2}\right)\right)\right|\right] \\
& \leq\left(\int_{0}^{\frac{1}{2}} t d t\right)^{1-\frac{1}{q}}\left(\int_{0}^{\frac{1}{2}} t\left|\psi^{\prime}\left(t\left(\frac{3 u-v}{2}\right)+(1-t)\left(\frac{3 v-u}{2}\right)\right)\right|^{q} d t\right)^{\frac{1}{q}}
\end{aligned}
$$




$$
\begin{gathered}
\leq\left(\frac{1}{8}\right)^{\frac{q-1}{q}}\left(\left[\frac{1}{2^{s+2}(s+2)}\right]\left|\psi^{\prime}\left(\frac{3 u-v}{2}\right)\right|^{q}\right. \\
\left.+\left[\frac{4-s 2^{-s}-3 \cdot 2^{-s}}{4((s+1)(s+2))}\right]\left|\psi^{\prime}\left(\frac{3 v-u}{2}\right)\right|^{q}\right)^{\frac{1}{q}} .
\end{gathered}
$$

Equivalently, we obtain

$$
\begin{aligned}
& \int_{\frac{1}{2}}^{1}(1-t)\left|\psi^{\prime}\left(\frac{3 v-u}{2}+2(u-v) t\right)\right| d t \\
& \leq\left(\frac{1}{8}\right)^{1-\frac{1}{q}}\left(\left[\frac{4-s 2^{-s}-3 \cdot 2^{-s}}{4((s+1)(s+2))}\right]\left|\psi^{\prime}\left(\frac{3 u-v}{2}\right)\right|^{q}\right. \\
& \left.\quad+\left[\frac{1}{2^{s+2}(s+2)}\right]\left|\psi^{\prime}\left(\frac{3 v-u}{2}\right)\right|^{q}\right)^{\frac{1}{q}} .
\end{aligned}
$$

Hence, from the inequalities (12), (15) and (16) we get the following:

$$
\begin{aligned}
& \left|\frac{1}{v-u} \int_{u}^{v} \psi(x) d x-\psi\left(\frac{u+v}{2}\right)\right| \\
& \quad \leq(v-u)\left(\frac{1}{8}\right)^{\frac{q-1}{q}}\left[\left(\frac{2-2^{-s}}{2(s+1)(s+2)}\right)\left[\left|\psi^{\prime}\left(\frac{3 u-v}{2}\right)\right|^{q}+\left|\psi^{\prime}\left(\frac{3 v-u}{2}\right)\right|^{q}\right]\right]^{\frac{1}{q}} \\
& \quad \leq(v-u)\left(\frac{1}{8}\right)^{\frac{q-1}{q}}\left[\frac{2-2^{-s}}{2(s+1)(s+2)}\right]^{\frac{1}{q}}\left[\left|\psi^{\prime}\left(\frac{3 u-v}{2}\right)\right|^{q}+\left|\psi^{\prime}\left(\frac{3 v-u}{2}\right)\right|^{q}\right]^{\frac{1}{q}}
\end{aligned}
$$

completing the proof of this theorem.

Corollary 1 Setting $s=1$ in Theorem 3, we get

$$
\left|\frac{1}{v-u} \int_{u}^{v} \psi(x) d x-\psi\left(\frac{u+v}{2}\right)\right| \leq \frac{v-u}{8}\left(\left|\psi^{\prime}\left(\frac{3 u-v}{2}\right)\right|^{q}+\left|\psi^{\prime}\left(\frac{3 u-v}{2}\right)\right|^{q}\right)^{\frac{1}{q}}
$$

Thus, Theorem 3 is a generalization of Theorem 2 .

Using a different approach, we get the result given in the next theorem.

Theorem 4 Let $\psi: E \subseteq \mathbb{R} \rightarrow \mathbb{R}$ be a differentiable mapping on $E^{\circ}, u, v \in E^{\circ}$ with $u \leq$ $v$, and its derivative $\psi^{\prime}:\left[\frac{3 u-v}{2}, \frac{3 v-u}{2}\right] \rightarrow \mathbb{R}$ a continuous function on $\left[\frac{3 u-v}{2}, \frac{3 v-u}{2}\right]$. If $\left|\psi^{\prime}\right|^{q}$ has $s$-convexity on $\left[\frac{3 u-v}{2}, \frac{3 v-u}{2}\right]$, for some fixed $0<s<1$, and $q \geq 1$, we obtain the following inequality:

$$
\begin{aligned}
& \left|\frac{1}{v-u} \int_{u}^{v} \psi(x) d x-\psi\left(\frac{u+v}{2}\right)\right| \\
& \quad \leq(v-u)\left(\frac{1}{8}\right)^{\frac{q}{1-q}}\left[\frac{1}{2^{s} \cdot 4(s+1)(s+2)}\right]^{\frac{1}{q}}\left[(s+1)^{\frac{1}{q}}+\left(2^{s} \cdot 4-s-3\right)^{\frac{1}{q}}\right]^{\frac{1}{q}} \\
& \quad \times\left(\left|\psi^{\prime}\left(\frac{3 u-v}{2}\right)\right|+\left|\psi^{\prime}\left(\frac{3 v-u}{2}\right)\right|\right) .
\end{aligned}
$$


Proof In view of (15), (16) and (12) we have

$$
\begin{aligned}
\mid \frac{1}{v-u} & \int_{u}^{v} \psi(x) d x-\psi\left(\frac{u+v}{2}\right) \mid \\
\leq & (v-u)\left(\frac{1}{8}\right)^{1-\frac{1}{q}}\left(\left[\frac{1}{2^{s+2}(s+2)}\right]\left|\psi^{\prime}\left(\frac{3 u-v}{2}\right)\right|^{q}\right. \\
& \left.+\left[\frac{4-s 2^{-s}-3 \cdot 2^{-s}}{4((s+1)(s+2))}\right]\left|\psi^{\prime}\left(\frac{3 v-u}{2}\right)\right|^{q}\right)^{\frac{1}{q}} \\
& +\left(\left[\frac{4-s 2^{-s}-3 \cdot 2^{-s}}{4((s+1)(s+2))}\right]\left|\psi^{\prime}\left(\frac{3 u-v}{2}\right)\right|^{q}+\left[\frac{1}{2^{s+2}(s+2)}\right]\left|\psi^{\prime}\left(\frac{3 v-u}{2}\right)\right|^{q}\right)^{\frac{1}{q}} \\
\leq & (v-u)\left(\frac{1}{8}\right)^{1-\frac{1}{q}}\left[\frac{1}{2^{s} \cdot 4(s+1)(s+2)}\right]^{\frac{1}{q}}\left((s+1)\left|\psi^{\prime}\left(\frac{3 u-v}{2}\right)\right|^{q}\right. \\
& +\left(2^{s} \cdot 4-s-3\right)\left|\psi^{\prime}\left(\frac{3 v-u}{2}\right)\right|^{q}+\left(2^{s} \cdot 4-s-3\right)\left|\psi^{\prime}\left(\frac{3 u-v}{2}\right)\right|^{q} \\
& \left.+(s+1)\left|\psi^{\prime}\left(\frac{3 v-u}{2}\right)\right|^{q}\right)^{\frac{1}{q}} \cdot
\end{aligned}
$$

Let $u_{1}=(s+1)\left|\psi^{\prime}\left(\frac{3 u-v}{2}\right)\right|^{q}, v_{1}=\left(2^{s} \cdot 4-s-3\right)\left|\psi^{\prime}\left(\frac{3 v-u}{2}\right)\right|^{q}, u_{2}=\left(2^{s} \cdot 4-s-3\right)\left|\psi^{\prime}\left(\frac{3 u-v}{2}\right)\right|^{q}$ and $v_{2}=(s+1)\left|\psi^{\prime}\left(\frac{3 v-u}{2}\right)\right|^{q}$.

Here, $0<\frac{1}{q}<1$, for $q \geq 1$, we use the fact

$$
\sum_{j=1}^{n}\left(u_{j}+v_{j}\right)^{r} \leq \sum_{j=1}^{n} u_{j}^{r}+\sum_{j=1}^{n} v_{j}^{r}
$$

where $0 \leq r<1, u_{1}, u_{2}, \ldots, u_{n} \geq 0$ and $v_{1}, v_{2}, \ldots, v_{n} \geq 0$, we get

$$
\begin{aligned}
& \left|\frac{1}{v-u} \int_{u}^{v} \psi(x) d x-\psi\left(\frac{u+v}{2}\right)\right| \\
& \leq(v-u)\left(\frac{1}{8}\right)^{\frac{q}{1-q}}\left[\frac{1}{2^{s} \cdot 4(s+1)(s+2)}\right]^{\frac{1}{q}} \\
& \left.\quad \times\left(\left((s+1)^{\frac{1}{q}}+\left(\left(2^{s} \cdot 4\right)-s-3\right)^{\frac{1}{q}}\right)\right)\left|\psi^{\prime}\left(\frac{3 u-v}{2}\right)\right|^{q}+\left|\psi^{\prime}\left(\frac{3 v-u}{2}\right)\right|^{q}\right)^{\frac{1}{q}} \\
& \leq(b-a)\left(\frac{1}{8}\right)^{\frac{q}{1-q}}\left[\frac{1}{2^{s} \cdot 4(s+1)(s+2)}\right]^{\frac{1}{q}}\left[(s+1)^{\frac{1}{q}}+\left(\left(2^{s} \cdot 4\right)-s-3\right)^{\frac{1}{q}}\right]^{\frac{1}{q}} \\
& \quad \times\left(\left|\psi^{\prime}\left(\frac{3 u-v}{2}\right)\right|+\left|\psi^{\prime}\left(\frac{3 v-u}{2}\right)\right|\right)
\end{aligned}
$$

completing the proof of Theorem 4.

Under some conditions applied on the function $\left|\psi^{\prime}\right|$, the numerical and analytical comparison between inequalities (5) and (10) was performed as an example.

Example 1 Suppose that $[u, v]=\left[\frac{-3}{4}, \frac{-1}{2}\right], s=\frac{1}{2}$ and $\psi(x)=e^{x}$. Let the second part of inequalities (5) and (10) be indicated by $I$ and $J$, respectively, then we get $J=-0.083435$ 
and $I=-0.02511936$ if $q=2$, and we have $J=-0.047584569$ and $I=-0.03450437$ if $q=1$. Therefore, the second part of inequality (10) is better than that of inequality (5) when $q=1$ and $q=2$.

Theorem 5 Suppose that $\psi: E^{\circ} \subset \mathbb{R} \rightarrow \mathbb{R}$ is a differentiable mapping on $E^{\circ}, u, v \in E^{\circ}$ with $u<v$. Let the derivative of $\psi$ be $\psi^{\prime}:\left[\frac{3 u-v}{2}, \frac{3 v-u}{2}\right] \rightarrow \mathbb{R}$, a continuous function on $\left[\frac{3 u-v}{2}, \frac{3 v-u}{2}\right]$. Let $q>1$, if $\left|\psi^{\prime}\right|^{q}$ has s-convexity on $\left[\frac{3 u-v}{2}, \frac{3 v-u}{2}\right]$, for some fixed $s \in(0,1)$, then we get the following inequality:

$$
\begin{aligned}
\left|\frac{1}{v-u} \int_{u}^{v} \psi(x) d x-\psi\left(\frac{u+v}{2}\right)\right| & \\
\leq & (v-u)\left(\frac{1}{2^{p+1}(p+1)}\right)^{\frac{1}{p}}\left[\frac{1}{2^{s+1}(s+1)}\right]^{\frac{1}{q}}\left(\left|\psi^{\prime}\left(\frac{3 u-v}{2}\right)\right|^{q}\right. \\
& \left.+\left[2^{s+1}-1\right]\left|\psi^{\prime}\left(\frac{3 v-u}{2}\right)\right|^{q}\right)^{\frac{1}{q}}+\left(\left[2^{s+1}-1\right]\left|\psi^{\prime}\left(\frac{3 u-v}{2}\right)\right|^{q}\right. \\
& \left.+\left|\psi^{\prime}\left(\frac{3 v-u}{2}\right)\right|^{q}\right)^{\frac{1}{q}},
\end{aligned}
$$

with $\frac{1}{p}+\frac{1}{q}=1$.

Proof Again, by the Hölder inequality, we get

$$
\begin{aligned}
& \int_{0}^{\frac{1}{2}} t\left|\psi^{\prime}\left(t\left(\frac{3 u-v}{2}\right)+(1-t)\left(\frac{3 v-u}{2}\right)\right)\right| \\
& \quad \leq\left[\int_{0}^{\frac{1}{2}} t^{p}\right]^{\frac{1}{p}}\left[\int_{0}^{\frac{1}{2}}\left|\psi^{\prime}\left(t\left(\frac{3 u-v}{2}\right)+(1-t)\left(\frac{3 v-u}{2}\right)\right)\right|^{q} d t\right]^{\frac{1}{q}} .
\end{aligned}
$$

Since the function $\left|\psi^{\prime}\right|$ is s-convex on $\left[\frac{3 u-v}{2}, \frac{3 v-u}{2}\right]$, we obtain

$$
\left|\psi^{\prime}\left(t\left(\frac{3 u-v}{2}\right)+(1-t) \frac{3 v-u}{2}\right)\right| \leq t^{s}\left|\psi^{\prime}\left(\frac{3 u-v}{2}\right)\right|+(1-t)^{s}\left|\psi^{\prime}\left(\frac{3 v-u}{2}\right)\right| \text {, }
$$

for any $t \in[0,1]$.

Therefore,

$$
\begin{aligned}
& {\left[\int_{0}^{\frac{1}{2}} t^{p}\right]^{\frac{1}{p}}\left[\int_{0}^{\frac{1}{2}}\left|\psi^{\prime}\left(t\left(\frac{3 u-v}{2}\right)+(1-t)\left(\frac{3 v-u}{2}\right)\right)\right|^{q} d t\right]^{\frac{1}{q}}} \\
& \leq\left[\frac{1}{(p+1) 2^{p+1}}\right]^{\frac{1}{p}}\left[\left|\psi^{\prime}\left(\frac{3 u-v}{2}\right)\right|^{q} \int_{0}^{\frac{1}{2}} t^{s} d t\right. \\
& \left.\quad+\left|\psi^{\prime}\left(\frac{3 v-u}{2}\right)\right|^{q} \int_{0}^{\frac{1}{2}}(1-t)^{s} d t\right]^{\frac{1}{q}} \\
& \leq\left[\frac{1}{(p+1) 2^{p+1}}\right]^{\frac{1}{p}}\left(\left[\frac{1}{2^{s+1}(s+1)}\right]\left|\psi^{\prime}\left(\frac{3 u-v}{2}\right)\right|^{q}\right. \\
& \left.\quad+\left[\frac{1-2^{-(s+1)}}{s+1}\right]\left|\psi^{\prime}\left(\frac{3 v-u}{2}\right)\right|^{q}\right)^{\frac{1}{q}} .
\end{aligned}
$$


Similarly, we get

$$
\begin{gathered}
\int_{\frac{1}{2}}^{1}(1-t)\left|\psi^{\prime}\left(t\left(\frac{3 u-v}{2}\right)+(1-t)\left(\frac{3 v-u}{2}\right)\right)\right| \\
\leq\left[\frac{1}{(p+1) 2^{p+1}}\right]^{\frac{1}{p}}\left(\left|\psi^{\prime}\left(\frac{3 u-v}{2}\right)\right|^{q}\left[\frac{1-2^{-s-1}}{s+1}\right]\right. \\
\left.+\left|\psi^{\prime}\left(\frac{3 v-u}{2}\right)\right|^{q}\left[\frac{1}{2^{s+1}(s+1)}\right]\right)^{\frac{1}{q}} .
\end{gathered}
$$

Finally, from (12), (19) and (20) we obtain the desired result.

Theorem 6 Let $\psi: I \subseteq \mathbb{R} \rightarrow \mathbb{R}$ be a differentiable mapping on $I^{0}, u, v \in I^{0}$ with $u<v$, and its derivative $\psi^{\prime}:\left[\frac{3 u-v}{2}, \frac{3 v-u}{2}\right] \rightarrow \mathbb{R}$ a continuous function on $\left[\frac{3 u-v}{2}, \frac{3 v-u}{2}\right]$. Let $q>1$, if $\left|\psi^{\prime}\right|^{q}$ is an s-convex function on $\left[\frac{3 u-v}{2}, \frac{3 v-u}{2}\right]$, then the following inequality holds:

$$
\begin{aligned}
& \left|\frac{1}{v-u} \int_{u}^{v} \psi(x) d x-\psi\left(\frac{u+v}{2}\right)\right| \\
& \leq(v-u)\left(\frac{1}{2^{p+1}(p+1)}\right)^{\frac{1}{p}}\left[\frac{1}{2^{s+1}(s+1)}\right]^{\frac{1}{q}}\left[\left(2^{s+1}-1\right)^{\frac{1}{q}}+1\right] \\
& \quad \times\left(\left|\psi^{\prime}\left(\frac{3 u-v}{2}\right)\right|+\left|\psi^{\prime}\left(\frac{3 v-u}{2}\right)\right|\right),
\end{aligned}
$$

with $\frac{1}{p}+\frac{1}{q}=1$

Proof We consider inequality (18) given as

$$
\begin{aligned}
& \left|\frac{1}{v-u} \int_{u}^{v} \psi(x) d x-\psi\left(\frac{u+v}{2}\right)\right| \\
& \leq(v-u)\left(\frac{1}{2^{p+1}(p+1)}\right)^{\frac{1}{p}}\left[\frac{1}{2^{s+1}(s+1)}\right]^{\frac{1}{q}} \\
& \quad \times\left(\left|\psi^{\prime}\left(\frac{3 u-v}{2}\right)\right|^{q}+\left[2^{s+1}-1\right]\left|\psi^{\prime}\left(\frac{3 v-u}{2}\right)\right|^{q}\right)^{\frac{1}{q}} \\
& \quad+\left(\left[2^{s+1}-1\right]\left|\psi^{\prime}\left(\frac{3 u-v}{2}\right)\right|^{q}+\left|\psi^{\prime}\left(\frac{3 v-u}{2}\right)\right|^{q}\right)^{\frac{1}{q}} .
\end{aligned}
$$

Let $u_{1}=\left|\psi^{\prime}\left(\frac{3 u-v}{2}\right)\right|^{q}, v_{1}=\left[2^{s+1}-1\right]\left|\psi^{\prime}\left(\frac{3 v-u}{2}\right)\right|^{q}, u_{2}=\left[2^{s+1}-1\right]\left|\psi^{\prime}\left(\frac{3 u-v}{2}\right)\right|^{q}$ and $v_{2}=\left|\psi^{\prime}\left(\frac{3 v-u}{2}\right)\right|^{q}$. Here, $0<\frac{1}{q}<1$, for $q \geq 1$. Using the fact

$$
\sum_{j=1}^{n}\left(u_{j}+v_{j}\right)^{r} \leq \sum_{j=1}^{n} u_{j}^{r}+\sum_{j=1}^{n} v_{j}^{r}
$$


whereby $0 \leq r<1, u_{1}, u_{2}, \ldots, u_{n} \geq 0$ and $v_{1}, v_{2}, \ldots, v_{n} \geq 0$, we obtain

$$
\begin{aligned}
& \left|\frac{1}{v-u} \int_{u}^{v} \psi(x) d x-\psi\left(\frac{u+v}{2}\right)\right| \\
& \leq(v-u)\left(\frac{1}{2^{p+1}(p+1)}\right)^{\frac{1}{p}}\left[\frac{1}{2^{s+1}(s+1)}\right]^{\frac{1}{q}}\left[\left(2^{s+1}-1\right)^{\frac{1}{q}}+1\right] \\
& \quad \times\left(\left|\psi^{\prime}\left(\frac{3 u-v}{2}\right)\right|+\left|\psi^{\prime}\left(\frac{3 v-u}{2}\right)\right|\right) .
\end{aligned}
$$

A variant of the Hermite-Hadamard-type inequality involving a first derivative for powers in terms is given as follows.

Theorem 7 Suppose that $\psi: E^{\circ} \subset \mathbb{R} \rightarrow \mathbb{R}$ is a differentiable mapping on $E^{\circ}$, such that $\psi^{\prime} \in L^{1}([u, v]), u, v \in E^{\circ}$ with $u<v$. Let $q \geq 1$, if $\left|\psi^{\prime}\right|^{q}$ is s-convex on $\left[\frac{3 u-v}{2}, \frac{3 v-u}{2}\right]$ for some fixed $s \in(0,1)$ and $q \geq 1$, then we get the following inequality:

$$
\begin{gathered}
\left|\frac{\psi\left(\frac{3 u-v}{2}\right)+\psi\left(\frac{3 v-u}{2}\right)+2 \psi\left(\frac{u+v}{2}\right)}{2}-\frac{1}{v-u} \int_{u}^{v} \psi(x) d x\right| \\
\leq \frac{v-u}{2}\left(\frac{1}{2}\right)^{\frac{q-1}{q}}\left[\frac{s+\left(\frac{1}{s}\right)^{s}}{(s+1)(s+2)}\right]^{\frac{1}{q}} \\
\quad \times\left(\left|\psi^{\prime}\left(\frac{3 u-v}{2}\right)\right|^{q}+\left|\psi^{\prime}\left(\frac{3 v-u}{2}\right)\right|^{q}\right)^{\frac{1}{q}} .
\end{gathered}
$$

Proof Applying Lemma 1, we obtain

$$
\begin{aligned}
& \left|\frac{\psi\left(\frac{3 u-v}{2}\right)+\psi\left(\frac{3 v-u}{2}\right)}{2}-\frac{1}{2(v-u)} \int_{\frac{3 u-v}{2}}^{\frac{3 v-u}{2}} \psi(x) d x\right| \\
& \quad \leq(v-u) \int_{0}^{1}|1-2 t|\left|\psi^{\prime}\left(t\left(\frac{3 u-v}{2}\right)+(1-t)\left(\frac{3 v-u}{2}\right)\right)\right| d t .
\end{aligned}
$$

From (8) and (23), we have

$$
\begin{aligned}
& \left|\frac{\psi\left(\frac{3 u-v}{2}\right)+\psi\left(\frac{3 v-u}{2}\right)+2 \psi\left(\frac{u+v}{2}\right)}{2}-\frac{1}{v-u} \int_{u}^{v} \psi(x) d x\right| \\
& \quad \leq \frac{(v-u)}{2} \int_{0}^{1}|1-2 t|\left|\psi^{\prime}\left(t\left(\frac{3 u-v}{2}\right)+(1-t)\left(\frac{3 v-u}{2}\right)\right)\right| d t .
\end{aligned}
$$

In the first case, we take $q=1$. We know that $\left|\psi^{\prime}\right|$ is $s$-convex on $\left[\frac{3 u-v}{2}, \frac{3 v-u}{2}\right]$, we get

$$
\begin{aligned}
& \left|\psi^{\prime}\left(t\left(\frac{3 u-v}{2}\right)+(1-t) \frac{3 v-u}{2}\right)\right| \\
& \quad \leq t^{s}\left|\psi^{\prime}\left(\frac{3 u-v}{2}\right)\right|+(1-t)^{s}\left|\psi^{\prime}\left(\frac{3 v-u}{2}\right)\right|,
\end{aligned}
$$

for any $t \in[0,1]$. 
Therefore,

$$
\begin{aligned}
\int_{0}^{1} & |1-2 t|\left[t^{s}\left|\psi^{\prime}\left(\frac{3 u-v}{2}\right)\right|+(1-t)^{s}\left|\psi^{\prime}\left(\frac{3 v-u}{2}\right)\right|\right] d t \\
= & \int_{0}^{1}\left[\left|\psi^{\prime}\left(\frac{3 u-v}{2}\right)\right| t^{s}|1-2 t|+\left|\psi^{\prime}\left(\frac{3 v-u}{2}\right)\right|(1-t)^{s}|1-2 t|\right] d t \\
= & {\left[\int_{0}^{\frac{1}{2}}\left[\left|\psi^{\prime}\left(\frac{3 u-v}{2}\right)\right| t^{s}(1-2 t)+\left|\psi^{\prime}\left(\frac{3 v-u}{2}\right)\right|(1-t)^{s}(1-2 t)\right] d t\right.} \\
& \left.+\int_{\frac{1}{2}}^{1}\left[\left|\psi^{\prime}\left(\frac{3 u-v}{2}\right)\right| t^{s}(2 t-1)+\left|\psi^{\prime}\left(\frac{3 v-u}{2}\right)\right|(1-t)^{s}(2 t-1)\right] d t\right] \\
= & \frac{1}{(s+1)(s+2)}\left(s+\frac{1}{2^{s}}\right)\left(\left|\psi^{\prime}\left(\frac{3 u-v}{2}\right)\right|+\left|\psi^{\prime}\left(\frac{3 v-u}{2}\right)\right|\right) .
\end{aligned}
$$

Combining the inequality (24) and (25), we get the inequality (22), which holds for $q=1$. Now in the second case, take $p=\frac{q}{q-1}$, when $q>1$. Using the well-known Hölder inequality we obtain

$$
\begin{aligned}
& \int_{0}^{1}|1-2 t|\left|\psi^{\prime}\left(t\left(\frac{3 u-v}{2}\right)+(1-t)\left(\frac{3 v-u}{2}\right)\right)\right| d t \\
& =\int_{0}^{1}|1-2 t|^{1-\frac{1}{q}}|1-2 t|^{\frac{1}{q}}\left|\psi^{\prime}\left(t\left(\frac{3 u-v}{2}\right)+(1-t)\left(\frac{3 v-u}{2}\right)\right)\right| d t \\
& \leq\left(\int_{0}^{1}|1-2 t| d t\right)^{\frac{q-1}{q}} \\
& \quad \times\left(\int_{0}^{1}|1-2 t| \mid \psi^{\prime}\left(t\left(\frac{3 u-v}{2}\right)+(1-t)\left(\frac{3 v-u}{2}\right)\right)^{q} d t\right)^{\frac{1}{q}} .
\end{aligned}
$$

From (24) and (26), we obtain

$$
\begin{aligned}
&\left|\frac{\psi\left(\frac{3 u-v}{2}\right)+\psi\left(\frac{3 v-u}{2}\right)+2 \psi\left(\frac{u+v}{2}\right)}{2}-\frac{1}{v-u} \int_{u}^{v} \psi(x) d x\right| \\
& \leq \frac{v-u}{2}\left(\int_{0}^{1}|1-2 t| d t\right)^{\frac{q-1}{q}} \\
& \quad \times\left(\int_{0}^{1}|1-2 t|\left|\psi^{\prime}\left(t\left(\frac{3 u-v}{2}\right)+(1-t)\left(\frac{3 v-u}{2}\right)\right)\right|^{q} d t\right)^{\frac{1}{q}} \\
& \leq \frac{v-u}{2}\left(\int_{0}^{1}|1-2 t| d t\right)^{\frac{q-1}{q}}\left[\frac{1}{(s+1)(s+2)}\left(s+\frac{1}{2^{s}}\right)\right]^{\frac{1}{q}} \\
& \times\left(\left|\psi^{\prime}\left(\frac{3 u-v}{2}\right)\right|^{q}+\left|\psi^{\prime}\left(\frac{3 v-u}{2}\right)\right|^{q}\right)^{\frac{1}{q}} \\
&= \frac{v-u}{2}\left(\frac{1}{2}\right)^{1-\frac{1}{q}}\left[\frac{\left(\frac{1}{s}\right)^{s}+s}{(s+1)(s+2)}\right]^{\frac{1}{q}} \\
& \times\left(\left|\psi^{\prime}\left(\frac{3 u-v}{2}\right)\right|^{q}+\left|\psi^{\prime}\left(\frac{3 v-u}{2}\right)\right|^{q}\right)^{\frac{1}{q}},
\end{aligned}
$$

completing the proof of Theorem 7. 
Theorem 8 Suppose that $\psi: E \subseteq \mathbb{R} \rightarrow \mathbb{R}$ is a differentiable mapping on $E^{\circ}, u, v \in E^{\circ}$ with $u \leq v$, and its derivative $\psi^{\prime \prime}:\left[\frac{3 u-v}{2}, \frac{3 v-u}{2}\right] \rightarrow \mathbb{R}$ is a continuous function on $\left[\frac{3 u-v}{2}, \frac{3 v-u}{2}\right]$. If $\left|\psi^{\prime \prime}\right|$ is an s-convex function on $\left[\frac{3 u-v}{2}, \frac{3 v-u}{2}\right]$, then the following inequality holds:

$$
\begin{aligned}
& \left|\frac{1}{v-u} \int_{0}^{1} \psi(x) d x-\frac{\psi\left(\frac{3 u-v}{2}\right)+\psi\left(\frac{3 v-u}{2}\right)+2 \psi\left(\frac{u+v}{2}\right)}{4}\right| \\
& \leq 2(v-u)^{2}\left[\frac{1}{(s+3)(s+2)}\left|\psi^{\prime \prime}\left(\frac{3 u-v}{2}\right)\right|\right. \\
& \left.\quad+\frac{1}{s+1}\left|\psi^{\prime \prime}\left(\frac{3 v-u}{2}\right)\right|\right] .
\end{aligned}
$$

Proof From Lemma 3, we get

$$
\begin{aligned}
& \frac{1}{2(v-u)} \int_{\frac{3 u-v}{2}}^{\frac{3 v-u}{2}} \psi(t) d t \\
& =\frac{\psi\left(\frac{3 v-u}{2}\right)+\psi\left(\frac{3 u-v}{2}\right)}{2} \\
& \quad-2(v-u)\left[\int_{0}^{1} t(1-t) \psi^{\prime \prime}\left(t \frac{3 u-v}{2}+(1-t) \frac{3 v-u}{2}\right) d t\right] .
\end{aligned}
$$

In view of inequalities (8) and (28), we obtain

$$
\begin{aligned}
& \left|\frac{1}{v-u} \int_{0}^{1} \psi(x) d x-\frac{\psi\left(\frac{3 u-v}{2}\right)+\psi\left(\frac{3 v-u}{2}\right)+2 \psi\left(\frac{u+v}{2}\right)}{4}\right| \\
& \quad \leq 2(v-u)^{2}\left[\int_{0}^{1} t(1-t)\left|\psi^{\prime \prime}\left(t\left(\frac{3 u-v}{2}\right)+(1-t) \psi^{\prime \prime}\left(\frac{3 v-u}{2}\right)\right)\right| d t\right] .
\end{aligned}
$$

Considering the fact that the given function $\left|\psi^{\prime \prime}\right|$ is $s$-convex on $\left[\frac{3 u-v}{2}, \frac{3 v-u}{2}\right]$, we have

$$
\left|\psi^{\prime \prime}\left(t\left(\frac{3 u-v}{2}\right)+(1-t) \frac{3 v-u}{2}\right)\right| \leq t^{s}\left|\psi^{\prime \prime}\left(\frac{3 u-v}{2}\right)\right|+(1-t)^{s}\left|\psi^{\prime \prime}\left(\frac{3 v-u}{2}\right)\right| .
$$

Therefore,

$$
\begin{aligned}
& \int_{0}^{1} t(1-t)\left|\psi^{\prime \prime}\left(t\left(\frac{3 u-v}{2}\right)+(1-t) \psi^{\prime \prime}\left(\frac{3 v-u}{2}\right)\right)\right| d t \\
& \quad \leq \int_{0}^{1}(1-t) t^{s+1} d t\left|\psi^{\prime \prime}\left(\frac{3 u-v}{2}\right)\right|+\int_{0}^{1}(1-t)^{s+1} d t\left|\psi^{\prime \prime}\left(\frac{3 v-u}{2}\right)\right| \\
& \quad \leq \frac{1}{(s+2)(s+3)}\left|\psi^{\prime \prime}\left(\frac{3 u-v}{2}\right)\right|+\frac{1}{s+2}\left|\psi^{\prime \prime}\left(\frac{3 v-u}{2}\right)\right| .
\end{aligned}
$$

By a simple computation,

$$
\begin{aligned}
& \int_{0}^{1}(1-t)^{s+1} d t=\frac{1}{s+2} \\
& \int_{0}^{1} t^{s+1}(1-t) d t=\frac{1}{(s+2)(s+3)} .
\end{aligned}
$$


Finally, from (29) and (30) we deduce the inequality (27) holds, completing the proof of Theorem 8.

The other type is obtained using the next theorem; the gamma function can be defined as follows:

$$
\Gamma(w)=\int_{0}^{\infty} t^{w-1} e^{-t} d t, \quad w>0 .
$$

Theorem 9 Suppose that $\psi: E \subseteq \mathbb{R} \rightarrow \mathbb{R}$ is a differentiable mapping on $E^{\circ}, u, v \in E^{\circ}$ with $u \leq v$, and its derivative $\psi^{\prime \prime}:\left[\frac{3 u-v}{2}, \frac{3 v-u}{2}\right] \rightarrow \mathbb{R}$, is a continuous function on $\left[\frac{3 u-v}{2}, \frac{3 v-u}{2}\right]$. Suppose that $q>1$, if $\left|\psi^{\prime \prime}\right|^{q}$ is an $s$-convex function on $\left[\frac{3 u-v}{2}, \frac{3 v-u}{2}\right]$, for some fixed $0<s<1$ with $\frac{1}{p}+\frac{1}{q}=1$, we have the following:

$$
\begin{gathered}
\left|\frac{1}{v-u} \int_{0}^{1} \psi(x) d x-\frac{\psi\left(\frac{3 u-v}{2}\right)+\psi\left(\frac{3 v-u}{2}\right)+2 \psi\left(\frac{u+v}{2}\right)}{4}\right| \\
\leq 2(v-u)^{2}\left(\frac{\sqrt{\pi} \Gamma(p+1)}{2^{1+2 p} \Gamma\left(p+\frac{3}{2}\right)}\right)^{\frac{1}{p}}\left(\frac{1}{s+1}\right)^{\frac{1}{q}} \\
\quad \times\left[\left|\psi^{\prime \prime}\left(\frac{3 u-v}{2}\right)\right|^{q}+\left|\psi^{\prime \prime}\left(\frac{3 v-u}{2}\right)\right|^{q}\right]^{\frac{1}{q}} \\
\leq 2(v-u)^{2}\left(\frac{\sqrt{\pi} \Gamma(p+1)}{2^{1+2 p} \Gamma\left(p+\frac{3}{2}\right)}\right)^{\frac{1}{p}} \\
\quad \times\left[\left|\psi^{\prime \prime}\left(\frac{3 u-v}{2}\right)\right|^{q}+\left|\psi^{\prime \prime}\left(\frac{3 v-u}{2}\right)\right|^{q}\right]^{\frac{1}{q}} .
\end{gathered}
$$

Proof Since $\left|\psi^{\prime \prime}\right|^{q}$ is $s$-convex, we obtain

$$
\left|\psi^{\prime \prime}(t a+(1-t) v)\right|^{q} \leq t^{s}\left|\psi^{\prime \prime}(u)\right|^{q}+(1-t)^{s}\left|\psi^{\prime \prime}(u)\right|^{q} .
$$

Using both (32) and the Hölder inequality, we get

$$
\begin{aligned}
& \int_{0}^{1}\left(t-t^{2}\right)\left|\psi^{\prime \prime}\left(t\left(\frac{3 u-v}{2}\right)+(1-t) \psi^{\prime \prime}\left(\frac{3 v-u}{2}\right)\right)\right| d t \\
& \leq \int_{0}^{1}\left(t-t^{2}\right)\left|\psi^{\prime \prime}\left(t\left(\frac{3 u-v}{2}\right)+(1-t) \psi^{\prime \prime}\left(\frac{3 v-u}{2}\right)\right)\right| d t \\
& \leq\left[\int_{0}^{1}\left(t-t^{2}\right)^{p}\right]^{\frac{1}{p}}\left[\left|\psi^{\prime \prime}\left(t\left(\frac{3 u-v}{2}\right)+(1-t) \psi^{\prime \prime}\left(\frac{3 v-u}{2}\right)\right)\right|^{q} d t\right]^{\frac{1}{q}} \\
& \leq {\left[\int_{0}^{1}\left(t-t^{2}\right)^{p}\right]^{\frac{1}{p}} } \\
& \times\left[\int_{0}^{1} t^{s} d t\left[\left|\psi^{\prime \prime}\left(\frac{3 u-v}{2}\right)\right|^{q}+\int_{0}^{1}(1-t)^{s} d t\left|\psi^{\prime \prime}\left(\frac{3 v-u}{2}\right)\right|^{q}\right]^{\frac{1}{q}}\right. \\
& \leq\left(\frac{\sqrt{\pi} \Gamma(p+1)}{2^{1+2 p} \Gamma\left(p+\frac{3}{2}\right)}\right)^{\frac{1}{p}}\left(\frac{1}{s+1}\right)^{\frac{1}{q}}\left[\left|\psi^{\prime \prime}\left(\frac{3 u-v}{2}\right)\right|^{q}+\left|\psi^{\prime \prime}\left(\frac{3 v-u}{2}\right)\right|^{q}\right]^{\frac{1}{q}} .
\end{aligned}
$$


In view of inequalities (29) and (33), we have the first inequality in (31). Now, for the second inequality in (31) we use the following facts: $\left(\frac{1}{s+1}\right)^{\frac{1}{q}} \leq 1, s \in(0,1]$ and $q>1$, completing the proof.

\section{Corollary 2}

$$
\begin{aligned}
& \left|\frac{1}{v-u} \int_{0}^{1} \psi(x) d x-\frac{\psi\left(\frac{3 u-v}{2}\right)+\psi\left(\frac{3 v-u}{2}\right)+2 \psi\left(\frac{u+v}{2}\right)}{4}\right| \\
& \quad \leq 2(v-u)^{2}\left(\frac{\sqrt{\pi} \Gamma(p+1)}{2^{1+2 p} \Gamma\left(p+\frac{3}{2}\right)}\right)^{\frac{1}{p}}\left[\left|\psi^{\prime \prime}\left(\frac{3 u-v}{2}\right)\right|^{q}+\left|\psi^{\prime \prime}\left(\frac{3 v-u}{2}\right)\right|^{q}\right]^{\frac{1}{q}} .
\end{aligned}
$$

Proof This results from Theorem 9, using $\left(\frac{1}{s+1}\right)^{\frac{1}{q}} \leq 1, s \in(0,1]$ and $q>1$.

A different Hermite-Hadamard-type inequality involving a second derivatives for powers in the terms is given as follows.

Theorem 10 Suppose that $f: E \subseteq \mathbb{R} \rightarrow \mathbb{R}$ is a differentiable mapping on $E^{\circ}, u, v \in E^{\circ}$ with $u \leq v$, and its derivative $\psi^{\prime \prime}:\left[\frac{3 u-v}{2}, \frac{3 v-u}{2}\right] \rightarrow \mathbb{R}$ is a continuous function on $\left[\frac{3 u-v}{2}, \frac{3 v-u}{2}\right]$. If $\left|\psi^{\prime \prime}\right|$ is an s-convex function on $\left[\frac{3 u-v}{2}, \frac{3 v-u}{2}\right]$, for $q>1$ and for some fixed $0<s \leq 1$ with $\frac{1}{p}+\frac{1}{q}=1$, we obtain the following:

$$
\begin{aligned}
& \left|\frac{1}{v-u} \int_{0}^{1} \psi(x) d x-\frac{\psi\left(\frac{3 u-v}{2}\right)+\psi\left(\frac{3 v-u}{2}\right)+2 \psi\left(\frac{u+v}{2}\right)}{4}\right| \\
& \leq 2(v-u)^{2}\left(\frac{1}{p+1}\right)^{\frac{1}{p}} \\
& \quad \times\left[\frac{\Gamma(s+1) \Gamma(q+1)}{\Gamma(q+s+2)}\left|\psi^{\prime \prime}\left(\frac{3 u-v}{2}\right)\right|^{q}+\frac{1}{q+s+1}\left|\psi^{\prime \prime}\left(\frac{3 v-u}{2}\right)\right|^{q}\right]^{\frac{1}{q}} .
\end{aligned}
$$

Proof From (29) and the Hölder inequality, we get

$$
\begin{aligned}
\int_{0}^{1}( & \left.t-t^{2}\right)\left|\psi^{\prime \prime}\left(t\left(\frac{3 u-v}{2}\right)+(1-t) \psi^{\prime \prime}\left(\frac{3 v-u}{2}\right)\right)\right| d t \\
\leq & {\left[\int_{0}^{1} t^{p} d t\right]^{\frac{1}{p}}\left[\int_{0}^{1}(1-t)^{q} \mid \psi^{\prime \prime}\left(t\left(\frac{3 u-v}{2}\right)\right.\right.} \\
& \left.\left.+(1-t) \psi^{\prime \prime}\left(\frac{3 v-u}{2}\right)\right)\left.\right|^{q} d t\right]^{\frac{1}{q}} \\
\leq & {\left[\int_{0}^{1} t^{p} d t\right]^{\frac{1}{p}}\left[\int_{0}^{1} t^{s}(1-t)^{q} d t\left|\psi^{\prime \prime}\left(\frac{3 u-v}{2}\right)\right|^{q}\right.} \\
& \left.+\int_{0}^{1}(1-t)^{s}(1-t)^{q} d t\left|\psi^{\prime \prime}\left(\frac{3 v-u}{2}\right)\right|^{q}\right]^{\frac{1}{q}} \\
\leq & \left(\frac{1}{p+1}\right)^{\frac{1}{p}}\left[B(s+1, q+1)\left|\psi^{\prime \prime}\left(\frac{3 u-v}{2}\right)\right|^{q}\right. \\
& \left.+\frac{1}{q+s+1}\left|\psi^{\prime \prime}\left(\frac{3 v-u}{2}\right)\right|^{q}\right]^{\frac{1}{q}}
\end{aligned}
$$




$$
\begin{aligned}
\leq & \left(\frac{1}{p+1}\right)^{\frac{1}{p}}\left[\frac{\Gamma(s+1) \Gamma(q+1)}{\Gamma(q+s+2)}\left|\psi^{\prime \prime}\left(\frac{3 u-v}{2}\right)\right|^{q}\right. \\
& \left.+\frac{1}{q+s+1}\left|\psi^{\prime \prime}\left(\frac{3 v-u}{2}\right)\right|^{q}\right]^{\frac{1}{q}}
\end{aligned}
$$

where

$$
B(s+1, s+1)=2^{1-2(s+1)} B\left(\frac{1}{2}, s+1\right)=2^{1-2(s+1)} \frac{\Gamma\left(\frac{1}{2}\right) \Gamma(s+1)}{\Gamma\left(\frac{s+3}{2}\right)} .
$$

Theorem 11 Suppose that $\psi: E \subseteq \mathbb{R} \rightarrow \mathbb{R}$ is a differentiable mapping on $E^{\circ}, u, v \in E^{\circ}$ with $u \leq v$, and its derivative $\psi^{\prime \prime}:\left[\frac{3 u-v}{2}, \frac{3 v-u}{2}\right] \rightarrow \mathbb{R}$ is a continuous function on $\left[\frac{3 u-v}{2}, \frac{3 v-u}{2}\right]$. If $\left|\psi^{\prime \prime}\right|$ is an s-convex function on $\left[\frac{3 u-v}{2}, \frac{3 v-u}{2}\right]$, for $q>1$ and for some fixed $0<s \leq 1$ with $\frac{1}{p}+\frac{1}{q}=1$, we obtain

$$
\begin{gathered}
\left|\frac{1}{v-u} \int_{0}^{1} \psi(x) d x-\frac{\psi\left(\frac{3 u-v}{2}\right)+\psi\left(\frac{3 v-u}{2}\right)+2 \psi\left(\frac{u+v}{2}\right)}{4}\right| \\
\leq\left(\frac{1}{2}\right)^{\frac{1}{p}}\left[\frac{\Gamma(s+2) \Gamma(q+1)}{\Gamma(q+s+3)}\left|\psi^{\prime \prime}\left(\frac{3 u-v}{2}\right)\right|^{q}\right. \\
\left.+\frac{\Gamma(2) \Gamma(q+s+1)}{\Gamma(q+s+3)}\left|\psi^{\prime \prime}\left(\frac{3 v-u}{2}\right)\right|^{q}\right]^{\frac{1}{q}} .
\end{gathered}
$$

Proof In similar way, we use the Hölder inequality and (29), to obtain the inequality

$$
\begin{aligned}
& \int_{0}^{1}\left(t-t^{2}\right)\left|\psi^{\prime \prime}\left(t\left(\frac{3 u-v}{2}\right)+(1-t) \psi^{\prime \prime}\left(\frac{3 v-u}{2}\right)\right)\right| d t \\
&=\int_{0}^{1} t^{1-\frac{1}{q}} d t\left[\int_{0}^{1} t^{\frac{1}{q}}(1-t) \mid \psi^{\prime \prime}\left(t\left(\frac{3 u-v}{2}\right)\right.\right. \\
&\left.\left.\quad+(1-t) \psi^{\prime \prime}\left(\frac{3 v-u}{2}\right)\right) \mid d t\right] \\
& \leq {\left[\int_{0}^{1} t d t\right]^{1-\frac{1}{q}}\left[\left|\psi^{\prime \prime}\left(\frac{3 u-v}{2}\right)\right|^{q} \int_{0}^{1} t^{s+1}(1-t)^{q} d t\right.} \\
&\left.+\left|\psi^{\prime \prime}\left(\frac{3 v-u}{2}\right)\right|^{q} \int_{0}^{1} t(1-t)^{q+s} d t\right]^{\frac{1}{q}} \\
& \leq\left(\frac{1}{2}\right)^{\frac{1}{p}}\left[\left|\psi^{\prime \prime}\left(\frac{3 u-v}{2}\right)\right|^{q} B(s+2, q+1)+\left|\psi^{\prime \prime}\left(\frac{3 v-u}{2}\right)\right|^{q} B(2, q+s+1)\right]^{\frac{1}{q}} \\
& \leq\left(\frac{1}{2}\right)^{\frac{1}{p}}\left[\left|\psi^{\prime \prime}\left(\frac{3 u-v}{2}\right)\right|^{q} \frac{\Gamma(s+2) \Gamma(q+1)}{\Gamma(q+s+3)}\right. \\
&\left.+\left|\psi^{\prime \prime}\left(\frac{3 v-u}{2}\right)\right|^{q} \frac{\Gamma(2) \Gamma(q+s+1)}{\Gamma(q+s+3)}\right]^{\frac{1}{q}} \cdot
\end{aligned}
$$

\section{Applications}

\subsection{Applications to special means}

Recalling the means of two non-negative numbers $u$ and $v$, we consider the following: 
1. The arithmetic mean:

$$
A=A(u, v)=\frac{u+v}{2} ; \quad u, v \in \mathbb{R}, \text { with } u, v>0 .
$$

2. The geometric mean:

$$
G=G(u, v)=\sqrt{u v} ; \quad u, v \in \mathbb{R} \text {, with } u, v>0 .
$$

3. The logarithmic mean:

$$
L(u, v)=\frac{v-u}{\log v-\log u} ; \quad u, v \in \mathbb{R} \text {, with } u, v>0 .
$$

4. The generalized logarithmic mean:

$$
L_{m}(u, v)=\left[\frac{v^{m+1}-u^{u+1}}{(v-u)(m+1)}\right]^{\frac{1}{m}} ; \quad m \in \mathbb{Z} \backslash\{-1,0\}, u, v \in \mathbb{R} \text {, with } u, v>0
$$

Using the results obtained in Sect. 2, and the above applications of the means, we get the following proposition.

Proposition 1 Suppose that $m \in \mathbb{Z} \backslash\{-1,0\}$ and $u, v \in \mathbb{R}$ such that $0<u<v$, we get the following:

$$
\begin{aligned}
& \left|A^{m}(u, v)-L_{m}^{m}(u, v)\right| \\
& \quad \leq(v-u)|m|\left(\frac{1}{8}\right)^{1-\frac{1}{q}}\left[\frac{2-2^{-s}}{2(s+1)(s+2)}\right]\left[A\left(\left|\frac{3 u-v}{2}\right|^{(m-1) q},\left|\frac{3 v-u}{2}\right|^{(m-1) q}\right)\right]^{\frac{1}{q}} .
\end{aligned}
$$

Proof This follows from Lemma 4 and Theorem 3, which apply for $\psi(x)=x^{m}$ and $m$ as specified above.

Proposition 2 Suppose that $m \in \mathbb{Z} \backslash\{-1,0\}$ and $u, v \in \mathbb{R}$ such that $0<u<v$, we obtain the following:

$$
\begin{aligned}
& \left|G^{-2}(u, v)-A^{-2}(u, v)\right| \\
& \quad \leq(v-u)|m|\left(\frac{1}{8}\right)^{1-\frac{1}{q}}\left[\frac{2-2^{-s}}{2(s+1)(s+2)}\right]\left[A\left(\left|\frac{3 u-v}{2}\right|^{-3 q},\left|\frac{3 v-u}{2}\right|^{-3 q}\right)\right]^{\frac{1}{q}} .
\end{aligned}
$$

Proof This follows from Lemma 4 and Theorem 3, which apply for $\psi(x)=\frac{1}{x^{2}}$.

Proposition 3 Let $m \in \mathbb{Z} \backslash\{-1,0\}$ and $u, v \in \mathbb{R}$ such that $0<u<v$, we get the following:

$$
\begin{aligned}
& \left|A^{-1}(u, v)-L^{-1}(u, v)\right| \\
& \quad \leq(v-u)\left(\frac{1}{8}\right)^{1-\frac{1}{q}}\left[\frac{2-2^{-s}}{2(s+1)(s+2)}\right]\left[A\left(\left|\frac{3 u-v}{2}\right|^{-2 q},\left|\frac{3 v-u}{2}\right|^{-2 q}\right)\right]^{\frac{1}{q}} .
\end{aligned}
$$

Proof This follows from Lemma 4 and Theorem 3, which apply for $\psi(x)=\frac{1}{x}$. 


\subsection{The midpoint formula}

Let $h$ be a partition $u=z_{0}<z_{1}<\cdots<z_{m-1}<z_{m}=v$ of the interval $[u, v]$; we consider the following quadrature formula:

$$
\int_{u}^{v} \psi(x) d x=T_{j}(\psi, h)+E_{j}(\psi, h), \quad j=1,2
$$

where

$$
T_{1}(\psi, h)=\sum_{j=0}^{m-1} \frac{\psi\left(z_{j}\right)+\psi\left(z_{j+1}\right)}{2}\left(z_{j+1}-z_{j}\right)
$$

given from its trapezoidal version, and

$$
T_{2}(\psi, h)=\sum_{j=0}^{m-1} \psi\left(\frac{z_{j}+z_{j+1}}{2}\right)\left(z_{j+1}-z_{j}\right)
$$

is for the midpoint version. The associated approximation error is denoted by $E_{j}(\psi, h)$.

Proposition 4 Let the function $\psi$ be an s-convex, for every partition of $\left[\frac{3 u-v}{2}, \frac{3 v-2}{2}\right]$ the midpoint error satisfies

$$
\begin{aligned}
& \left|\int_{u}^{v} \psi(x) d x+E_{2}(\psi, h)\right| \\
& \quad \leq \sum_{i=0}^{m-1}\left(z_{i+1}-z_{i}\right) \frac{\left|\psi\left(\frac{3 z_{i}-z_{i+1}}{2}\right)+\psi\left(\frac{3 z_{i+1}-z_{i}}{2}\right)\right|}{2(s+1)} \\
& \quad \leq \frac{1}{s+1} \sum_{i=1}^{m-1}\left(z_{i+1}-z_{i}\right) \max \left[\left(\left|\psi\left(\frac{3 z_{i}-z_{i+1}}{2}\right), \psi\left(\frac{3 z_{i+1}-z_{i}}{2}\right)\right|\right)\right] .
\end{aligned}
$$

Proof From Lemma 4, we obtain

$$
\left|2 \int_{z_{i}}^{z_{i+1}} \psi(x) d x-\left(z_{i+1}-z_{i}\right) \psi\left(\frac{z_{i}+z_{i+1}}{2}\right)\right| \leq\left(z_{i+1}-z_{i}\right) \frac{\left|\psi\left(\frac{3 z_{i}-z_{i+1}}{2}\right)+\psi\left(\frac{3 z_{i+1}-z_{i}}{2}\right)\right|}{2(s+1)} .
$$

On the other hand, we have

$$
\begin{aligned}
& \left|\int_{u}^{v} \psi(x) d x+\left[\int_{u}^{v} \psi(x) d x-T_{2}(\psi, h)\right]\right| \\
& \quad=\left|\sum_{j=0}^{m-1}\left[2 \int_{z_{j}}^{z_{j+1}} \psi(x) d x-\left(z_{j+1}-z_{j}\right) \psi\left(\frac{z_{j}+z_{j+1}}{2}\right)\right]\right| \\
& \quad \leq \sum_{j=0}^{m-1}\left(z_{j+1}-z_{j}\right) \frac{\left|\psi\left(\frac{3 z_{j}-z_{j+1}}{2}\right)+\psi\left(\frac{3 z_{j+1}-z_{j}}{2}\right)\right|}{2(s+1)} \\
& \quad \leq \frac{1}{s+1} \sum_{j=1}^{m-1}\left(z_{j+1}-z_{j}\right) \max \left[\left(\left|\psi\left(\frac{3 z_{j}-z_{j+1}}{2}\right), \psi\left(\frac{3 z_{j+1}-z_{j}}{2}\right)\right|\right)\right] .
\end{aligned}
$$


Proposition 5 Suppose that the function $|\psi|^{q}, q \geq 1$, is an s-convex, for every partition of $\left[\frac{3 u-v}{2}, \frac{3 v-2}{2}\right]$ the midpoint error satisfies

$$
\begin{aligned}
& \left|\int_{u}^{v} \psi(x) d x+E_{2}(\psi, h)\right| \\
& \quad \leq \sum_{j=0}^{m-1}\left(z_{j+1}-z_{j}\right) \frac{\left|\psi\left(\frac{3 z_{j}-z_{j+1}}{2}\right)+\psi\left(\frac{3 z_{j+1}-z_{j}}{2}\right)\right|}{2(s+1)} \\
& \quad \leq \frac{1}{s+1} \sum_{j=1}^{m-1}\left(z_{j+1}-z_{j}\right) \max \left[\left(\left|\psi\left(\frac{3 z_{j}-z_{j+1}}{2}\right)+\psi\left(\frac{3 z_{j+1}-z_{j}}{2}\right)\right|\right)\right] .
\end{aligned}
$$

Proof From Theorem 3, we obtain

$$
\begin{aligned}
& \left|\int_{z_{j}}^{z_{j+1}} \psi(x) d x-\left(z_{j+1}-z_{j}\right) \psi\left(\frac{z_{j}+z_{j+1}}{2}\right)\right| \\
& \quad \leq\left(\frac{1}{8}\right)^{1-\frac{1}{q}}\left[\frac{2-2^{-s}}{2(s+1)(s+2)}\right]^{\frac{1}{q}}\left(z_{j+1}-z_{j}\right)^{2} \\
& \quad \times\left[\left|\psi\left(\frac{3 z_{j}-z_{j+1}}{2}\right)\right|^{q}+\left.\psi\left(\frac{3 z_{j+1}-z_{j}}{2}\right)\right|^{q}\right]^{\frac{1}{q}} .
\end{aligned}
$$

Alternatively, we have

$$
\begin{aligned}
& \left|\int_{u}^{v} \psi(x) d x-T_{2}(\psi, h)\right| \\
& =\left|\sum_{j=0}^{m-1}\left[\int_{z_{j}}^{z_{j+1}} \psi(z) d z-\left(z_{j+1}-z_{j}\right) \psi\left(\frac{z_{j}+z_{j+1}}{2}\right)\right]\right| \\
& \leq \quad\left(\frac{1}{8}\right)^{\frac{q}{1-q}}\left[\frac{2-2^{-s}}{2(s+1)(s+2)}\right]^{\frac{1}{q}} \\
& \quad \times \sum_{j=0}^{m-1}\left(z_{j+1}-z_{j}\right)^{2}\left[\left|\psi\left(\frac{3 z_{j}-z_{j+1}}{2}\right)\right|^{q}+\left.\psi\left(\frac{3 z_{j+1}-z_{j}}{2}\right)\right|^{q}\right]^{\frac{1}{q}} \\
& \leq\left(\frac{1}{8}\right)^{\frac{q}{1-q}}\left[\frac{2-2^{-s}}{2(s+1)(s+2)}\right]^{\frac{1}{q}} \\
& \quad \times \sum_{j=1}^{m-1}\left(z_{j+1}-z_{j}\right)^{2} \max \left[\left(\left|\psi\left(\frac{3 z_{j}-z_{j+1}}{2}\right), \psi\left(\frac{3 z_{j+1}-z_{j}}{2}\right)\right|\right)\right] .
\end{aligned}
$$

Funding

Not applicable.

\section{Availability of data and materials}

Not applicable.

Competing interests

The authors declare that they have no competing interests. 


\section{Author details}

${ }^{1}$ Department of Mathematics, University of Hafr Al-Batin, Hafr Al-Batin, Saudi Arabia. ${ }^{2}$ Department of Mathematics and Institute for Mathematical Research, Universiti Putra Malaysia, Serdang, Malaysia.

\section{Publisher's Note}

Springer Nature remains neutral with regard to jurisdictional claims in published maps and institutional affiliations.

Received: 8 August 2019 Accepted: 9 October 2019 Published online: 17 October 2019

\section{References}

1. Yang, X.M.: On E-convex sets, E-convex functions, and E-convex programming. J. Optim. Theory Appl. 109(3), 699-704 (2001)

2. Xie, P., You, K., Tempo, R., Song, S., Wu, C.: Distributed convex optimization with inequality constraints over time-varying unbalanced digraphs. IEEE Trans. Autom. Control 63(12), 4331-4337 (2018)

3. Dragomir, S.S., Fitzpatrick, S.: The Hadamard inequalities for s-convex functions in the second sense. Demonstr. Math. 32(4), 687-696 (1999)

4. Özcan, S., Işcan, I.: Some new Hermite-Hadamard type inequalities for s-convex functions and their applications. J. Inequal. Appl. 2019(1), 201 (2019)

5. Dragomir, S.S., Pearce, C.: Selected topics on Hermite-Hadamard inequalities and applications. Math. Prepr. Arch. 2003(3), 463-817 (2003)

6. Mitrinović, D.S., Lacković, I.B.: Hermite and convexity. Aequ. Math. 28(1), 229-232 (1985)

7. Milovanović, G.V., Rassias, M.T.: Analytic number theory, approximation theory and special functions. In: Anderson, G.D., Vuorinen, M., Zhang, X. (eds.) Topics in Special Functions, vol. 3, pp. 297-345. Springer, Berlin (2014)

8. Hudzik, H., Maligranda, L.: Some remarks on s-convex functions. Aequ. Math. 48(1), 100-111 (1994)

9. Dragomir, S.S., Agarwal, R.P.: Two inequalities for differentiable mappings and applications to special means of real numbers and to trapezoidal formula. Appl. Math. Lett. 11(5), 91-95 (1998)

10. Özdemir, M.E.: A theorem on mappings with bounded derivatives with applications to quadrature rules and means. Appl. Math. Comput. 138(2-3), 425-434 (2003)

11. Kirmaci, U.S.: Inequalities for differentiable mappings and applications to special means of real numbers and to midpoint formula. Appl. Math. Comput. 147(1), 137-146 (2004)

12. Almutairi, A., Kılıçman, A.: New refinements of the Hadamard inequality on coordinated convex function. J. Inequal. Appl. 2019(1), 192 (2019)

13. Mehrez, K., Agarwal, P.: New Hermite-Hadamard type integral inequalities for convex functions and their applications. J. Comput. Appl. Math. 350, 274-285 (2019)

\section{Submit your manuscript to a SpringerOpen ${ }^{\circ}$ journal and benefit from:}

- Convenient online submission

- Rigorous peer review

- Open access: articles freely available online

- High visibility within the field

Retaining the copyright to your article

Submit your next manuscript at $\boldsymbol{~ s p r i n g e r o p e n . c o m ~}$ 